\title{
Quantification of multispectral photoacoustic images: unsupervised unmixing methods comparison
}

\author{
Aneline DOLET \\ 1. Univ.Lyon, INSA-Lyon, Université \\ Claude Bernard Lyon 1, UJM-Saint \\ Etienne, CNRS, Inserm, CREATIS \\ UMR 5220, U1206, F-69621, LYON, \\ France \\ 2. Department of Information \\ Engineering, University of Florence, \\ Florence, Italy \\ aneline.dolet@creatis.insa-lyon.fr \\ Cédric RICHARD \\ Laboratoire Lagrange, Université de \\ Nice Sophia-Antipolis, France \\ cedric.richard@unice.fr
}

\author{
Rita AMMANOUIL \\ Laboratoire Lagrange, Université de \\ Nice Sophia-Antipolis, France \\ rita.ammanouil@oca.eu \\ Piero TORTOLI \\ Department of Information \\ Engineering, University of Florence, \\ Florence, Italy \\ piero.tortoli@unifi.it
}

\author{
Thomas GRENIER \\ Univ.Lyon, INSA-Lyon, Université \\ Claude Bernard Lyon 1, UJM-Saint \\ Etienne, CNRS, Inserm, CREATIS \\ UMR 5220, U1206, F-69621, LYON, \\ France \\ thomas.grenier@creatis.insa-lyon.fr
}

Didier VRAY

Univ.Lyon, INSA-Lyon, Université

Claude Bernard Lyon 1, UJM-Saint

Etienne, CNRS, Inserm, CREATIS

UMR 5220, U1206, F-69621, LYON, France

didier.vray@creatis.insa-lyon.fr

\author{
François VARRAY \\ Univ.Lyon, INSA-Lyon, Université \\ Claude Bernard Lyon 1, UJM-Saint \\ Etienne, CNRS, Inserm, CREATIS \\ UMR 5220, U1206, F-69621, LYON, \\ France \\ francois.varray@creatis.insa-lyon.fr
}

\begin{abstract}
Multispectral photoacoustic imaging is a functional modality based on the detection of ultrasound waves coming from tissues illuminated by laser pulses at different wavelengths. The specific photoacoustic behavior of different media for each illuminating wavelength allows their quantification which is of great interest for various medical applications. The quantification algorithm performances are related to the correct estimation of reference spectra for each medium to quantify. This study aims at comparing three different unsupervised methods to extract these reference spectra (Group Lasso with Unit sum and Positivity constraints, Vertex Component Analysis and Spatio-Spectral Mean-Shift). After the reference extraction, a supervised unmixing method called Fully Constrained Least-Square is used to estimate the medium concentrations. Using Vevo LAZR as acquisition system, the quantification performances are evaluated on a colored $4 \%$ agar phantom containing two pure media and a mix of both. The results highlight the suitability of the Spatio-Spectral Mean-Shift to extract reference spectra that allow the assessment of photoacoustic for dataset medium concentration.
\end{abstract}

Keywords-photoacoustic imaging, unmixing algorithm, quantification

\section{INTRODUCTION}

Photoacoustic imaging is a promising modality to image and discriminate biological tissues illuminated by laser pulses. Optical absorbers have thermal expansions, due to the temperature increase following the optical energy absorption. For each transmitted laser pulse ultrasound waves are generated, propagate inside the tissue and can be detected on the tissue surface by a standard ultrasound probe. The optical absorption depends on the laser wavelength, so that each medium is characterized by a specific multispectral photoacoustic response, which allows its quantification [1]. More particularly, the wavelength range 600-900 $\mathrm{nm}$ allows the differentiation between oxygenated and deoxygenated blood which is of interest to follow-up diseases like carcinoma [2].

Classically, quantification methods are supervised and required reference spectra. The optimization of their estimation is still a tricky task marginally discussed in the literature. However, these references significantly impact on the quantification results. This study aims at comparing unsupervised methods able to extract the reference spectra from the data. Group Lasso with Unit sum and Positivity constraints (GLUP) [3] and Virtual Component Analysis (VCA) [4], typically used in hyperspectral fields, are compared to the Spatio-Spectral Mean-Shift (SSM-S) [5] already applied to cluster photoacoustic data.

\section{METHODS}

\section{A. Linear mixing model}

In multispectral photoacoustic imaging, the region of interest is imaged at different wavelengths. Each pixel is then characterized by a spectrum corresponding to the collection of the acquired intensity values at each wavelength. A pixel's spectrum is either pure, i.e. composed of a single pure medium, and then considered as an endmember (reference spectrum) or mixed, i.e. composed of a mixture of endmembers. 
Data unmixing requires solving a mixing model which can be linear or non-linear. In our context, the linear mixing model (LMM) has to be considered [6]. Such model assigns a mixed pixel to a convex combination of the endmembers. More formally, it can be defined for a single pixel $i \in[1 ; N]$, with $N$ the number of pixels of the dataset, by the following equation:

$$
\boldsymbol{A}_{i}=\sum_{j=1}^{k} u_{j i} \boldsymbol{E}_{j}+\boldsymbol{g}_{i}, \forall i \in[1 ; N]
$$

where $\boldsymbol{A}_{i}$ is the $L$-dimensional spectrum of the $i$-th pixel, $k$ the number of endmembers, $u_{j i}$ the abundance of the $j$-th endmember in the $i$-th pixel, $\boldsymbol{E}_{j}$ is the $L$-dimensional spectrum of the $j$-th endmember and $\boldsymbol{g}_{i}$ is a vector of Gaussian white noise accounting for sensor noise and error of the model. As they correspond to concentration, the coefficients $u_{j i}$ have to be under constraints of positivity and sum-to-one, as follow:

$$
\left\{\begin{array}{l}
u_{j i} \geq 0 \\
\sum_{j=1}^{k} u_{j i}=1
\end{array}\right.
$$

Even if LMM is a simple model, it well represents the studied unmixing model. It has also been largely used in remote sensing [6]. For medical applications, it can be a powerful tool for quantifying media in order to accurately assess the medium concentration.

\section{B. Reference spectra extraction}

The unmixing pipeline used in this study is based on two steps: (1) the endmembers extraction using unsupervised method and (2) the abundance estimation using a supervised method and the endmembers previously extracted. In this study, the comparison between methods is done only considering the first step which is the more critical. The abundance estimation is always conducted with the same strategy: Fully Constrained Least-Square [7]. Therefore, only the extracted endmembers will differ.

a) Group Lasso with Unit sum and Positivity constraints: The GLUP algorithm assumes that the endmembers are present in the image, among the observations [3]. With this assumption, LMM (1) can be reformulated as:

$$
\boldsymbol{A}_{i}=\sum_{j=1}^{N} c_{G_{j i}} \boldsymbol{A}_{j}+\boldsymbol{g}_{i}, \forall i \in[1 ; N]
$$

Like before, $c_{G_{j i}}$ is the abundance of $\boldsymbol{A}_{j}$ in $\boldsymbol{A}_{i}$. If $\boldsymbol{A}_{j}$ is an endmember, the row $\boldsymbol{C}_{G_{j}}$ of the abundance matrix has nonzero entries and represents the corresponding abundance map. By contrary, if $\boldsymbol{A}_{j}$ is a mixed pixel, $\boldsymbol{C}_{G_{j}}$ has all its elements equal to zero. In this way, $\boldsymbol{C}_{G}$ admits $N-k$ rows of zero, the other rows being equal to rows of $\boldsymbol{U}$. The assumption in GLUP is that $\boldsymbol{C}_{G}$ allows the identification of the endmembers in $\boldsymbol{A}$ through its non-zero rows. This property is exploited in GLUP in order to find the endmembers among the observations. The unmixing problem under investigation requires that $\boldsymbol{C}_{G}$ only has a few rows different from zero, in addition to the non-negativity and sum-to-one constraints which leads to the following convex optimization problem:

$$
\begin{gathered}
\min _{C_{G}}\left(\frac{1}{2} \sum_{j=1}^{N}\left\|\left(\boldsymbol{A}-\boldsymbol{A} \boldsymbol{C}_{G}\right)\right\|_{F}^{2}+\mu \sum_{j=1}^{N}\left\|\boldsymbol{C}_{G_{j}}\right\|_{2}\right) \\
\text { subject to }\left\{\begin{array}{c}
c_{G_{j i}} \geq 0 \forall i, j \\
\sum_{j=1}^{N} c_{G_{j i}}=1 \forall i
\end{array}\right.
\end{gathered}
$$

with $\mu \geq 0$ a regularization parameter and $\boldsymbol{A}$ the dataset matrix. The first term in (4) allows the matching of the observations with the model of (3), the second term induces sparsity by possibly driving several rows of $\boldsymbol{C}_{G}$ to zero [8]. The minimization is constrained to ensure that the abundances obey the positivity and the sum-to-one constraints. The resulting optimization is solved using a primal dual method explained in [3].

Even if GLUP also provides some estimated abundances corresponding to the non-zero rows in the estimated matrix $\boldsymbol{C}_{G}$, in the context of this study and in order to compare only the endmembers extraction methods, these abundances are not taken into account.

b) Vertex Component Analysis: VCA also assumes the presence of pure pixels in the data [4]. At each iteration of the algorithm, the data are projected onto a direction orthogonal to the subspace constituted from the endmembers already extracted. The farthest signal in this projection is then extracted as a new endmember. A new subspace, considering this endmember, is calculated and the same procedure is iteratively process until the extraction of the asked number of endmembers, i.e. $k$.

The initial considered subspace has first to be defined. It is calculated using two different methods depending on the dataset signal-to-noise ratio (SNR). If the SNR is superior to a threshold value $T$ (see [4] for its calculation), this first subspace is calculated using singular value decomposition (SVD) algorithm [9]. Otherwise, the considered subspace is constructed with the $\mathrm{k}-1$ first axes extracted by the Principal Component Analysis algorithm.

c) Spatio-Spectral Mean-Shift: This clustering algorithm based on a spatial and spectral regularization is largely presented in [5]. It assesses high clusterization performances on photoacoustic dataset and calculates for each created cluster a representative photoacoustic spectrum. For a particular cluster, the representative is the weighted mean of all the spectra included in the cluster. It is then possible to consider the representatives calculated by the SSM-S as extracted endmembers.

\section{Abundance estimation}

At this step, the endmembers are known and the abundances can be estimated using the Fully Constrained Least-Square (FCLS) [7]. This method takes into account the constraints of (2) which are required in our context. FCLS solves the following equation:

$$
\min _{C}\left(\frac{1}{2} \sum_{j=1}^{N}\|(\boldsymbol{A}-\boldsymbol{C} \boldsymbol{E})\|_{F}^{2}\right) \text { with }\left\{\begin{array}{c}
c_{j i} \geq 0 \forall i, j \\
\sum_{j=1}^{N} c_{j i}=1 \forall i
\end{array}\right.
$$

which is a similar problem to the GLUP one, presented in (4), with the regularization parameter $\mu=0$ and using the estimated endmembers matrix $\boldsymbol{E}$ rather than the dataset 
matrix $\boldsymbol{A}$. FCLS is then better conditioned than the problem of equation (4) because the endmembers are now known. Thus, it should lead to better abundance assessments than the GLUP one.

\section{MATERIALS}

\section{A. Imaged sample}

The imaged sample is a colored $4 \%$ agar phantom containing three different region of interest (Fig. 1): pure blue (left), pure green (right) and a mix of both (center). The blue concentration is 0.42 and the green one is 0.67 . The total concentration value of the mix is not exactly 1 because of the used pipette accuracy to measure ink doses.

\section{B. Photoacoustic acquisition system}

The commercial system Vevo LAZR (Visualsonics, Fujifilm) was used for multispectral photoacoustic acquisitions. The optical source was a Nd:YAG pulsed laser with pulse duration of $5 \mathrm{~ns}$ and $20 \mathrm{~Hz}$ repetition rate coupled with an optical parametric oscillator to access various wavelengths [10]. The acquisitions were based on the LZ400 probe (Fig. 1), composed of 256 elements that acquired ultrasound in the frequency range $18-38 \mathrm{MHz}$, while the used laser wavelength range was $[680 \mathrm{~nm} ; 820 \mathrm{~nm}]$ with $20 \mathrm{~nm}$ steps.

\section{RESULTS}

\section{A. Qualitative evaluation}

Fig. 2 shows the resulting abundance maps as well as the ground truth for qualitative comparison. For the blue abundance maps, the pure blue part (left) can be clearly seen only for VCA and SSM-S. The boundary between mix (center) and green (right) parts is however only visible with SSM-S. For the green abundance maps, all compared methods give close qualitative results: the blue part (left) is easily distinguishable and both other parts are more difficult to discriminate.
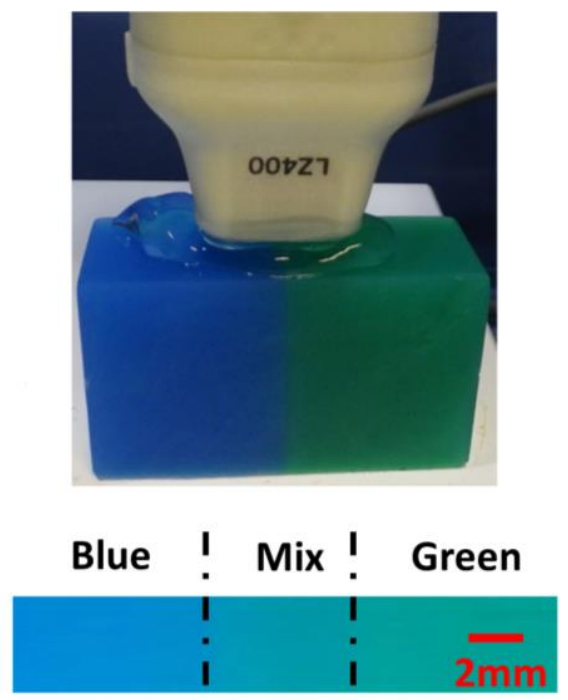

Fig. 1. Up - Phantom in the Vevo LAZR system with the LZ400 probe aligned with the region of interest for acquisitions. Down - Close up of the imaged region.

\section{B. Quantitative validation method}

In order to quantitatively compare the different tested methods, the average estimated abundances of the endmembers are calculated on the three phantom parts. For each endmember, the maximal average value is considered as corresponding to a pure phantom part. It allows a normalization of the average values by this maximal value. The estimated pure part has then a normalized mean abundance of 1 and the other parts have values between 0 and 1 .

Therefore, our quantitative validation expectations are: (1) to estimate the pure phantom part in the accurate location for both endmembers, (2) to estimate accurate average abundance values of the mix and (3) to estimate really low values for the absent medium in the pure phantom parts (e.g. blue abundance close to 0 considering the green endmember and the green phantom part).

\section{Quantitative validation evaluation}

The average estimated abundance values are summarized in Table 1. The ground truth, i.e. the expected values, are presented in the first three lines. The first expectation of pure medium accurate location, presented in the previous section, is then satisfied for all the methods.

Concerning the mix part, the GLUP algorithm assesses high blue abundance, even higher than the green estimated one for the mix, which have no sens considering the mix exact proportions. The VCA algorithm perfectly evaluates the blue abundance but overestimates the green one in the mix. The SSM-S algorithm allows the access to unperfect but accurate abundance values for both media in the mixture. This last algorithm achieves the best performances considering the evaluation of mix of media.

The lowest estimated abundance values of blue in pure green part, or vice-versa, is reached with SSM-S. Indeed, the maximal value is for green in the blue part and is of 0.1 which is really low. For the VCA algorithm, the calculated values are around 0.25 which could be acceptable but anyway higher than the SSM-S one. However, for GLUP, the value is acceptable for the blue part (0.11) but overestimated for the green one (0.62).

TABLE I. AVERAGE ESTIMATED ABUNDANCE VALUES

\begin{tabular}{|c|c|c|c|}
\hline & Phantom part & Blue map & Green map \\
\hline \multirow{3}{*}{ Ground truth } & Left & 1 & $\mathbf{0}$ \\
\hline & Center & 0.42 & 0.67 \\
\hline & Right & $\mathbf{0}$ & 1 \\
\hline \multirow{3}{*}{ GLUP } & Left & 1 & 0.11 \\
\hline & Center & 0.83 & 0.73 \\
\hline & Right & 0.62 & 1 \\
\hline \multirow{3}{*}{$\mathrm{VCA}$} & Left & 1 & 0.20 \\
\hline & Center & 0.42 & 0.89 \\
\hline & Right & 0.27 & 1 \\
\hline \multirow{3}{*}{ SSM-S } & Left & 1 & 0.02 \\
\hline & Center & 0.35 & 0.73 \\
\hline & Right & 0.10 & 1 \\
\hline
\end{tabular}




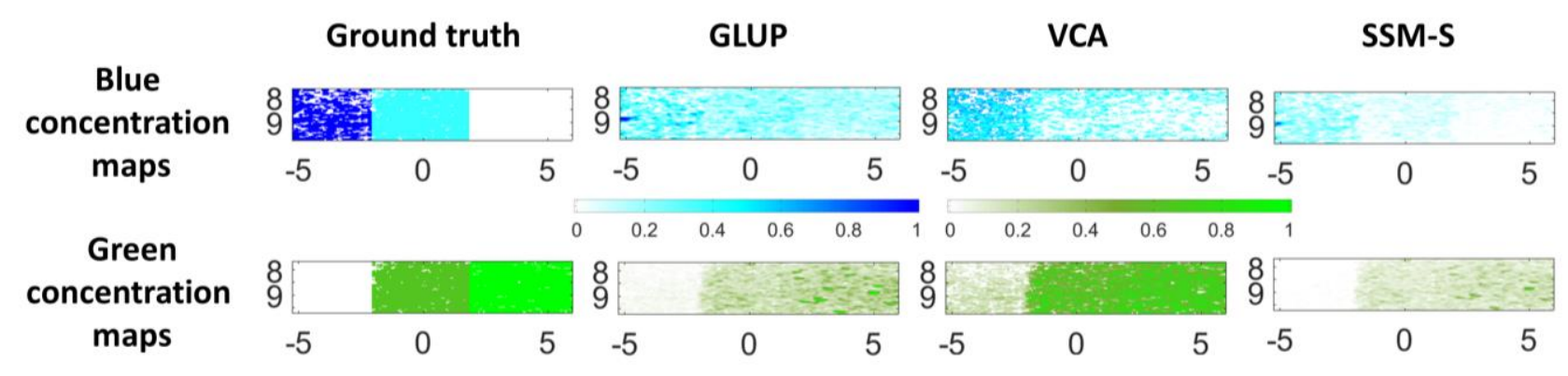

Fig. 2. Blue (up) and green (down) abundance maps calculated by the FCLS algorithm with the endmembers extracted by GLUP, VCA and the SSM-S algorithms (second, third and last columns, respectively). The ground truth is shown in the first column. The map axes are in mm.

Regarding the entire imaged region, the best performances are achieved by the combination of SSM-S algorithm, for the endmember extraction, with FCLS abundance estimation method.

\section{CONCLUSION AND DISCUSSION}

This study reports the comparison between three different unmixing pipelines for photoacoustic data unmixing. In each pipeline, two processing steps are applied to the data. The first one allows the endmembers extraction that corresponds to the calculation of reference spectra for each medium to quantify. The second processing step is the calculation of the abundance values that are the concentration. The comparisons done in this study are only about the endmembers extraction.

Three methods have been compared: GLUP, VCA and SSM-S. The results are qualitatively and quantitatively analysed. Qualitatively on the endmember abundance maps, the VCA and SSM-S algorithms seem to best evaluate the imaged region concentrations. The quantitative assessment is however more accurate and allows the validation of the SSM-S/FCLS pipeline which clearly achieves the best performances on all phantom parts.

However, all these methods assume that the endmembers are present in the dataset. In our phantom case, it is obviously the case but for many cases in biological tissues this assumption is really strong and probably not verified. Tests on biological tissue with precise ground truth are then necessary to validate the proposed pipeline in the photoacoustic field.

Finally, as similar results have been observed on other phantoms with various mix and dilutions, the SSM-S algorithm is anyway a powerful method to discriminate photoacoustic data [5] and extract endmembers. Using SSM-S/FCLS pipeline allows accurate estimation of medium concentration information which is of great interest for several medical applications.

\section{ACKNOWLEDGMENT}

This work was performed within the frameworks of LABEX CELYA (ANR-10-LABX-0060) and LABEX PRIMES (ANR-10-LABX-0063) of Université de Lyon, within the program "Investissements d'Avenir" (ANR-11-IDEX-0007) operated by the French National Research Agency (ANR). It was also supported by the Université Franco-Italienne (VINCI 2016) and the Région Rhône-Alpes (EXPLORA DOC). The authors also would like to thank the research council of Université Claude Bernard Lyon 1 for his financial support.

\section{REFERENCES}

[1] M. Vallet, F. Varray, J. Boutet, J.M. Dinten, G. Caliano, A.S. Savoia, and D. Vray, "Quantitative comparison of PZT and CMUT probes for photoacoustic imaging: experimental validation," Photoacoustics 8 , pp.48-58, 2017.

[2] D.R. Bauer, R. Olafsson, L.G. Montilla, and R.S. Witte, "In vivo multi-modality photoacoustic and pulse echo tracking of prostate tumor growth using a window chamber," in BiOS. International Society for Optics and Photonics, 2010, pp.75643B-75643B.

[3] R. Ammanouil, A. Ferrari, C. Richard and D. Mary, "Blind and fully constrained unmixing of hyperspectral images," IEEE Transactions on Image Processing, vol. 23, no. 12, pp. 5510-5518, 2014.

[4] J. M. P. Nascimento and J. M. B. Dias, "Vertex component analysis: a fast algorithm to unmix hyperspectral data", IEEE Transactions on Geoscience and Remote Sensing, vol. 43, pp. 898-910, 2004.

[5] A. Dolet, F. Varray, S. Mure, T. Grenier, Y. Liu, Z. Yuan, P. Tortoli and D. Vray, "Spatial and spectral regularization to discriminate tissues using multispectral photoacoustic imaging", EURASIP Journal on Advances in Signal Processing, vol. 39, no. 1, pp. 1-10, 2018 .

[6] N. Keshava and J. F. Mustard, "Spectral unmixing", IEEE Signal Processing Magazine, vol. 19, no. 1, pp. 44-57, 2002.

[7] D. C. Heinz and Chein-I-Chang, "Fully constrained least squares linear spectral mixture analysis method for material quantification in hyperspectral imagery", IEEE Transactions on Geoscience and Remote Sensing, vol. 39, no. 3, pp. 529-545, 2001.

[8] M. Yuan and Y. Lin, "Model selection and estimation in regression with grouped variables", Journal of the Royal Statistical Society: Series B (Statistical methodology), vol. 68, no. 1, pp. 49-67, 2006.

[9] L. L. Scharf, "Statistical signal processing: detection, estimation, and time series analysis", 1 Edition. Reading, Mass: Pearson, 1991.

[10] C. J. Arthuis, A. Novell, F. Raes, J. M. Escoffre, S. Lerondel, A. Le Pape, A. Bouakaz and F. Perrotin, "Real-time monitoring of placental oxygenation during maternal hypoxia and hyperoxygenation using photoacoustic imaging", PLOS ONE, vol. 12, no. 1, pp. 01698500169850-11, 2017. 